\title{
Transcatheter valve insertion in a model of enlarged right ventricular outflow tracts
}

\author{
Adeline Basquin, MD, Emmanuelle Pineau, MD, Louise Galmiche, MD, Damien Bonnet, MD, PhD, \\ Daniel Sidi, MD, PhD, and Younes Boudjemline, MD, PhD
}

\begin{abstract}
Objective: Transcatheter pulmonary valve insertion has recently emerged as an alternative to surgery. To extend its indications to patients with a large right ventricular outflow tract, we previously developed an intravascular device that reduces the diameter of the main pulmonary artery, allowing the insertion of available valved stents. Here we report its use in a model of animals with an enlarged right ventricular outflow tract and pulmonary valve incompetence.
\end{abstract}

\begin{abstract}
Methods and Results: The study comprised 33 sheep that first underwent surgical enlargement of the main pulmonary artery. We then intended to implant a filler percutaneously, followed later by the insertion of a valve. Three animals died during the intermediate stage. The remainder were humanely killed either immediately (group $1, \mathrm{n}=6$ ) or after a mean follow-up of 1 (group $2, \mathrm{n}=12$ ) or 2 months (group $3, \mathrm{n}=12$ ). Animals from groups 2 and 3 were equally divided into 2 subgroups according to the difference between diameters of the device inserted and the main pulmonary artery $(A<5 \mathrm{~mm}, B \geq 5 \mathrm{~mm}$ ). Fillers were all inserted successfully $(\mathrm{n}=30)$, although one embolized after its insertion (group 3A). A valved stent was implanted in all animals, but in 1 case a balloon ruptured during inflation of the stent leading to incomplete expansion and the death of the animal. Six animals, 5 of which were from group A, had pulmonary regurgitation after valve insertion.
\end{abstract}

Conclusion: Pulmonary valve insertion is possible through a transcatheter technique using a pulmonary artery filler. Oversizing the device reduces the risk of embolization and paraprosthetic leak. (J Thorac Cardiovasc Surg 2010;139:198-208)

Pulmonary valve incompetence (PVI), although generally well tolerated at short-term or midterm follow-up, has clearly been identified as a risk factor leading to significant longterm morbidity and mortality. ${ }^{1-3}$ In fact, it results in right ventricular (RV) dilatation, chronic heart failure, and ventricular arrhythmias. ${ }^{4-12}$ The only way to correct this insufficiency is to surgically replace the pulmonary valve (PV) ${ }^{9,12} \mathrm{~A}$ transcatheter technique to insert a PV has been developed. ${ }^{13-15}$ The only valve widely used and authorized for this indication in Europe is the Melody valve (Medtronic Inc, Minneapolis, Minn). Its use is presently limited to the small number of patients having a nonnative RV-pulmonary artery (PA) connection smaller than $22 \mathrm{~mm}$ in diameter. Patients operated on for tetralogy of Fallot represent most of the patients in need of a PV. They are often excluded from this technique because of the diameter of their right ventricular outflow tract (RVOT). As a result, these patients are only treatable by surgery. In parallel, off-pump PV insertion has been developed, ${ }^{16,17}$ but this approach still requires opening the

From the Service de Cardiologie Pédiatrie, Hôpital Necker-Enfants Malades, Assistance Publique Hôpitaux de Paris, and Faculté de Médecine Necker Enfants Malades, Université Paris V, Paris, France.

The study was supported by la Ville de Paris, Paris, France.

Received for publication Nov 24, 2008; revisions received May 30, 2009; accepted for publication July 8, 2009; available ahead of print Aug 26, 2009.

Address for reprints: Younes Boudjemline, MD, Service de Cardiologie Pédiatrique, Hôpital Necker-Enfants-Malades, 149, rue de Sèvres, 75015 Paris Cedex, France

(E-mail: younes.boudjemline@nck.ap-hop-paris.fr).

0022-5223/\$36.00

Copyright (c) 2010 by The American Association for Thoracic Surgery doi:10.1016/j.jtcvs.2009.07.025 chest. Over the past years, we and others have been working on a nonsurgical approach. $\mathrm{We}^{18}$ have already reported the use of a preshaped self-expandable stent forming a double cylinder. The external cylinder allowed anchoring to the dilated RVOT. The internal diameter was calibrated to authorize the fixation of commercially available valved stents. Several modifications of the original design were made to fit with the anatomy of patients in need of valve replacement. $\mathrm{We}^{19}$ recently reported the use of such a stent in animals. Although the design was promising, the delivery of the device remained complicated and needed multiple maneuvers at the time of deployment. These complications forced us to rethink the shape of the stent. Moreover, the stability of the stent had been tested in animals with a native RVOT. To test the stability in a larger RVOT, we conducted and report here an experimental study with optimized design of the PA filler in an ovine model with large and asymmetrical RVOTs.

\section{METHODS \\ PA Filler}

The filler is a self-expandable stent constructed from a single $0.22-\mathrm{mm}$ nitinol wire (AMF, Reuilly, France) in the shape of a cylinder with a double diameter. The extremities are directed inward and back toward an intermediate position forming a second wall in parallel with the external one. In comparison, in the previous design, the extremities were directed outward and back toward the middle part. This simple modification facilitates the delivery of the device, avoiding extra handling for its deployment (Figure 1). Besides the improvement of the delivery, the new configuration offers a larger surface of contact with the wall of the PA. To guarantee the sealing, we sutured a $0.3-\mathrm{mm}$ polytetrafluoroethylene (PTFE) membrane along the device with a 4-0 Prolene polypropylene thread (Ethicon, Inc, Somerville, 

Abbreviations and Acronyms
PA = pulmonary artery
PTFE = polytetrafluoroethylene
$\mathrm{PV}=$ pulmonary valve
PVI = pulmonary valve incompetence
$\mathrm{RV}=$ right ventricle (ventricular)
$\mathrm{RVOT}=$ right ventricular outflow tract

NJ). The device was available with various external diameters from 30 to 40 $\mathrm{mm}$ by increments of $5 \mathrm{~mm}$. The size of the device was chosen according to the grouping (see below). The overall lengths of the deployed and crimped device were, respectively, 20 and $35 \mathrm{~mm}$. The delivery system is a 20F Mullins sheath (Medtronic, Inc, Minneapolis, Minn). No balloons were required for its delivery.

\section{Preparation of the Valved Stent}

The valved stents are prepared as previously reported using bicuspid or tricuspid 22-mm valves harvested in a slaughterhouse from the bovine jugular vein. These valves are sutured in our laboratory with a 4-0 Ti-Cron thread (Covidien, Norwalk, Conn) into a balloon-expandable stent (CP stent; NuMED, Inc, Hopkinton, NY). Two lengths of CP stent are used: CP8Z34 and CP8Z28. Once prepared, valved stents are stored in a solution of glutaraldehyde. The delivery system used was very similar to the one developed by NuMED, Inc, for the insertion of the Melody valve (Ensemble). It was constituted by a $22-\mathrm{mm}$ balloon in a balloon catheter with a long sheath sliding over it. The distal part where the valved stent is crimped over the balloon was 20F. Beyond this initial 5-cm section, the catheter was $14 \mathrm{~F}$.

\section{Animals}

Thirty-three sheep weighing 60 to $75 \mathrm{~kg}$ were included in the study. We first intended to enlarge the RVOT and create PVI in all. After recovery, we planned for the animals to receive the PA filler immediately followed by the insertion of a valved stent through a transcatheter approach. All animals were treated according to European regulations for animal experimentation. The study was reviewed and approved by a local ethical committee. Survivors of the initial operation $(n=30)$ were divided into 3 groups according to the time when they were humanely killed. Operations in groups 1,2, and 3 were performed sequentially starting with group 1 . Six animals were included in a feasibility group and humanely killed just after filler and valve insertions (group 1). The remaining 24 animals were equally divided into 2 groups and humanely killed after a follow-up of 1 (group 2) or 2 months (group 3). In each of the 2 latter groups, animals were prospectively divided into 2 subgroups depending on the diameter of the implant to be inserted. Animals included in subgroup A received a filler 0 to $4 \mathrm{~mm}$ larger than the diameter of the PA, whereas animals included in subgroup B received a filler at least $5 \mathrm{~mm}$ larger than the measured PA diameter.

\section{Creation of the Model}

Anesthesia was induced with $10 \mathrm{mg} / \mathrm{kg}$ of thiopental and maintained with halothane $(1 \%-5 \%)$ under mechanical ventilation. A left thoracotomy was performed in the fourth intercostal space to expose the RVOT. The circumference of the PA was measured by sliding a noose around the PA. A pericardial patch was then prepared with the idea of constructing an RVOT of $40 \mathrm{~mm}$ in diameter (circumference $120 \mathrm{~mm}$ ) by $35 \mathrm{~mm}$ in length. A vascular clamp was positioned laterally on the main PA with the PV being roughly in one third of the incision. After 1 or 2 minutes of partial clamping to verify hemodynamic stability, an incision of $40 \mathrm{~mm}$ in length was performed. A leaflet of the PV was then excised. The prepared pericardial patch was thereafter stitched on the edges of the incision with a 5-0 Prolene polypropylene thread (Figure 2). Hemostasis was controlled by partial opening
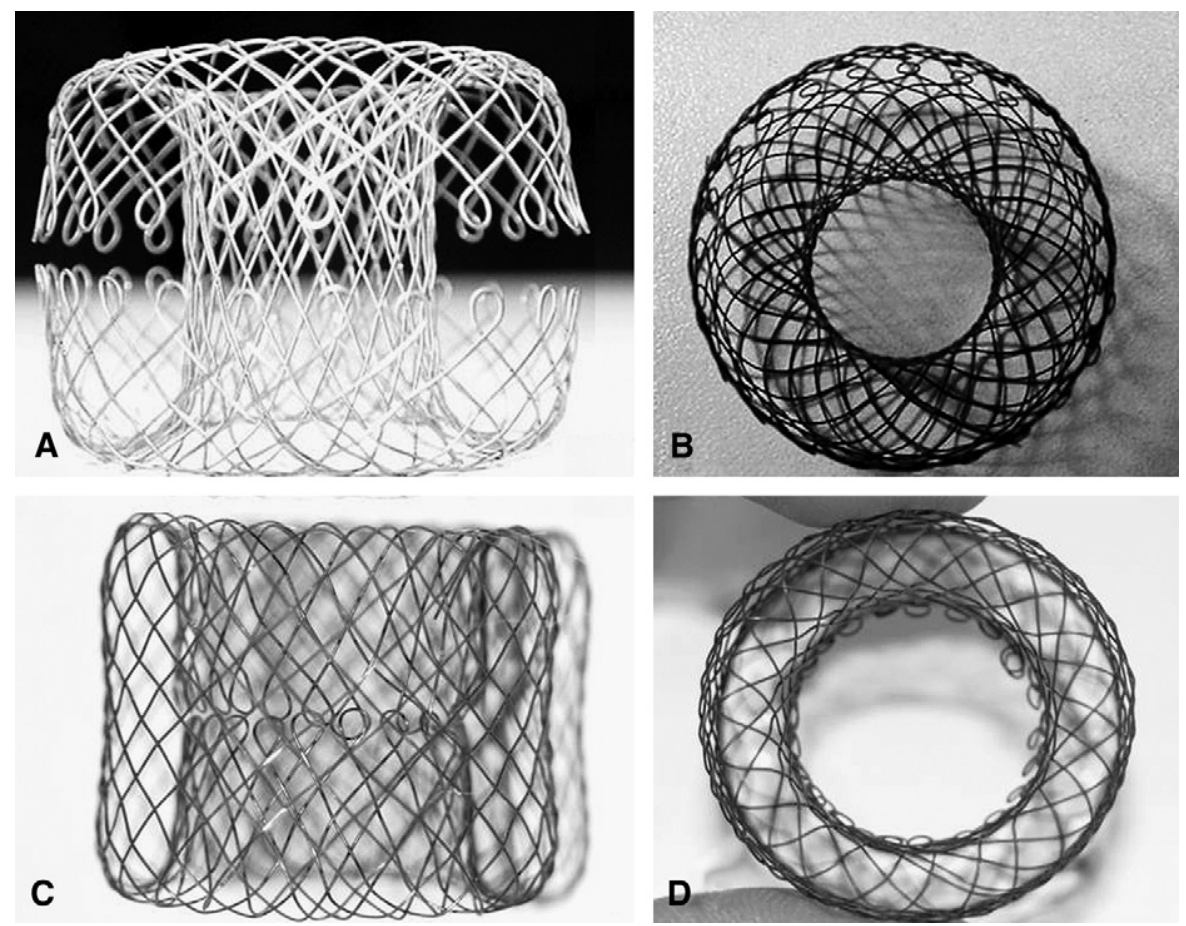

FIGURE 1. C and D, The newly designed stent is shown uncovered (front and lateral views) and compared with the old design on the top panels (A and B). The extremities are directed inward and back toward an intermediate position forming a second wall in parallel with the external one. In comparison, in the previous design, the extremities were directed outward and back toward the middle part. 

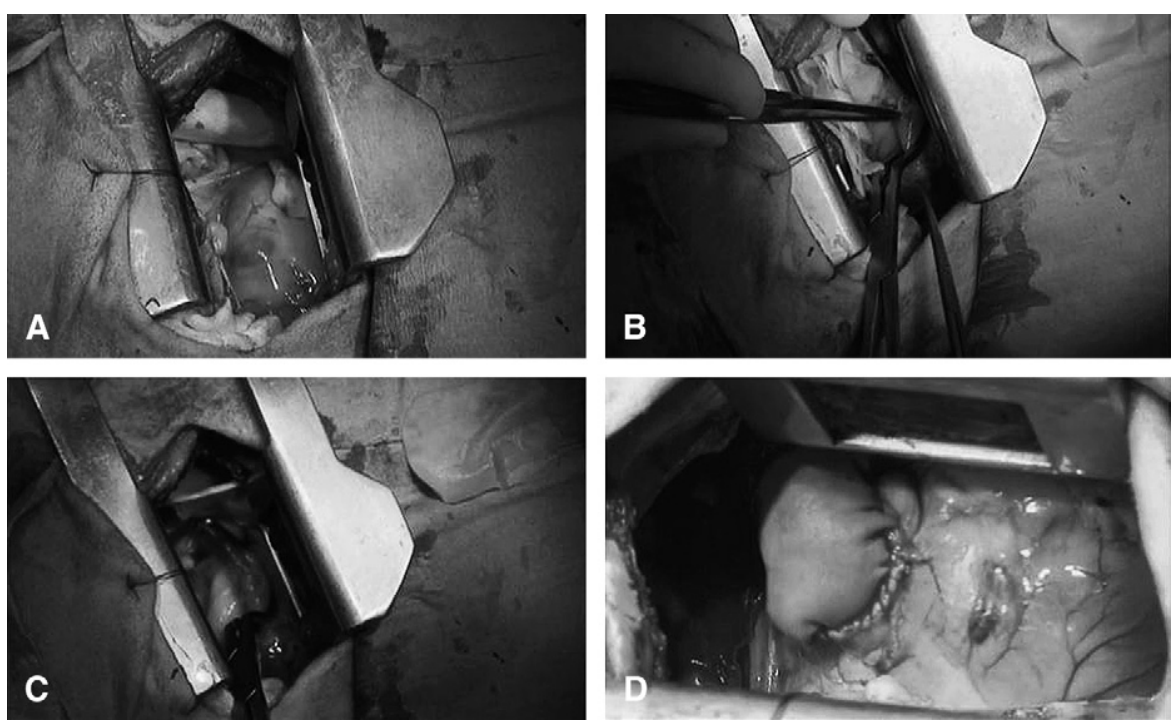

FIGURE 2. Surgical views showing the various steps of the enlargement of the RVOT. A, Incision through a left thoracotomy showing the RVOT with the PA at the top. B, Excision of a pulmonary valve leaflet after partial crossclamping of the PA. C, Sewing of the pericardial patch. D, Final aspect of the RVOT.

of the clamp. Additional stitches were placed if necessary to obtain complete sealing. When satisfactory, the clamp was completely removed and the thorax was closed over a thoracic drain. The latter was removed 1 hour after the operation. Tramadol and antibiotics were administrated to animals for 24 hours. The animals were then allowed to recover for at least 8 weeks.

\section{Insertion of the PA Filler}

Cardiac catheterization was performed under general anesthesia in mechanically ventilated sheep. Anesthesia was induced by an intravenous injection of thiopental, $10 \mathrm{mg} / \mathrm{kg}$, and was maintained with inhaled halothane. Cardiac and respiratory functions were monitored throughout the procedure. A sheath was placed in the right jugular vein. A 5F right coronary catheter (Cordis SA, France) was advanced in the distal PA. A stiff 0.035 -inch extrastiff guidewire was then placed through the catheter in the distal PA. A 5F catheter with radiopaque markers was used for hemodynamic assessment and dye injection. The PA was measured at the level of the aneurysm in both systole and diastole. For device selection, the maximum diameter was considered. Inflation of a compliant balloon (PTS; NuMED, Inc) was also used to appreciate the distensibility. After measurement and according to the subgrouping, a filler was selected and crimped in the delivery system. This latter was advanced over the wire in the enlarged RVOT. The delivery was achieved only by the simple tasks of removing the sheath and uncovering the device. No other maneuver was necessary to configure the device, which was placed at the level of the enlargement and proximal to the native valve (Figure 3). The delivery system was then removed, with the wire left in position.

\section{Insertion of the Valved Stent}

Immediately after the insertion of the filler, the valved stent was loaded into its delivery system and advanced over the same wire up to the PA. When in the central part of the filler, the valved stent was uncovered and balloons were subsequently inflated to deploy the valve. If necessary, its position was adjusted before the inflation of the second balloon. After deflation, the delivery system was removed. The wire was left in place until the final evaluation.

\section{Evaluation}

In the short-term group (group 1), we only assessed the feasibility of the filler insertion. No hemodynamic study was performed. In animals from groups 2 and 3, a complete hemodynamic study was performed using a marked catheter before the implantation procedure. Pressures in all right cavities were measured. Two different angiograms in the lateral view were done: one in the RV to assess its size and one in the PA to assess the anatomy and diameter of the PA and to look for PVI. To avoid unwanted mobilization of the filler, we made no measurements after its insertion. After valve insertion, the same measurements were repeated to quantify the gradient across the prosthesis, the sealing of the filler, and the presence of PVI. Cardiac catheterization was repeated each month until the animals were humanely killed. Paraprosthetic or prosthetic leakage was sought on the 2 different angiograms. "Proximal leakage" was defined as leakage observed during the RV angiogram whereas distal leakage was defined as leakage seen during the PA angiogram.

\section{Graft Retrieval}

The animals were humanely killed after complete assessment at the end point determined by the protocol. A left thoracotomy was performed and the lungs and heart were retrieved en bloc after a lethal injection of thiopental. The pulmonary vascular tree was inspected macroscopically, focusing on the pulmonary wall, device position, aspect of the pericardial patch, and the native PA valve. RVOTs were preserved in a formaldehyde solution. Specimens were embedded in methylmethacrylate. Various stains were performed (von Kossa, orcein, hematoxylin and eosin, and Masson's trichrome) to study in particular the interface between the arterial wall and the device. Histologic examination was performed only in animals from group 3 .

\section{Statistical Analysis}

Data are presented as mean and range. The Student paired $t$ test was used to evaluate the difference between subgroups A and B.

\section{RESULTS \\ Animal Model}

Thirty-three animals were operated on. Three animals died either at the time of surgery $(n=2)$ or before the second stage $(n=1)$. One died of a hemorrhage when the 

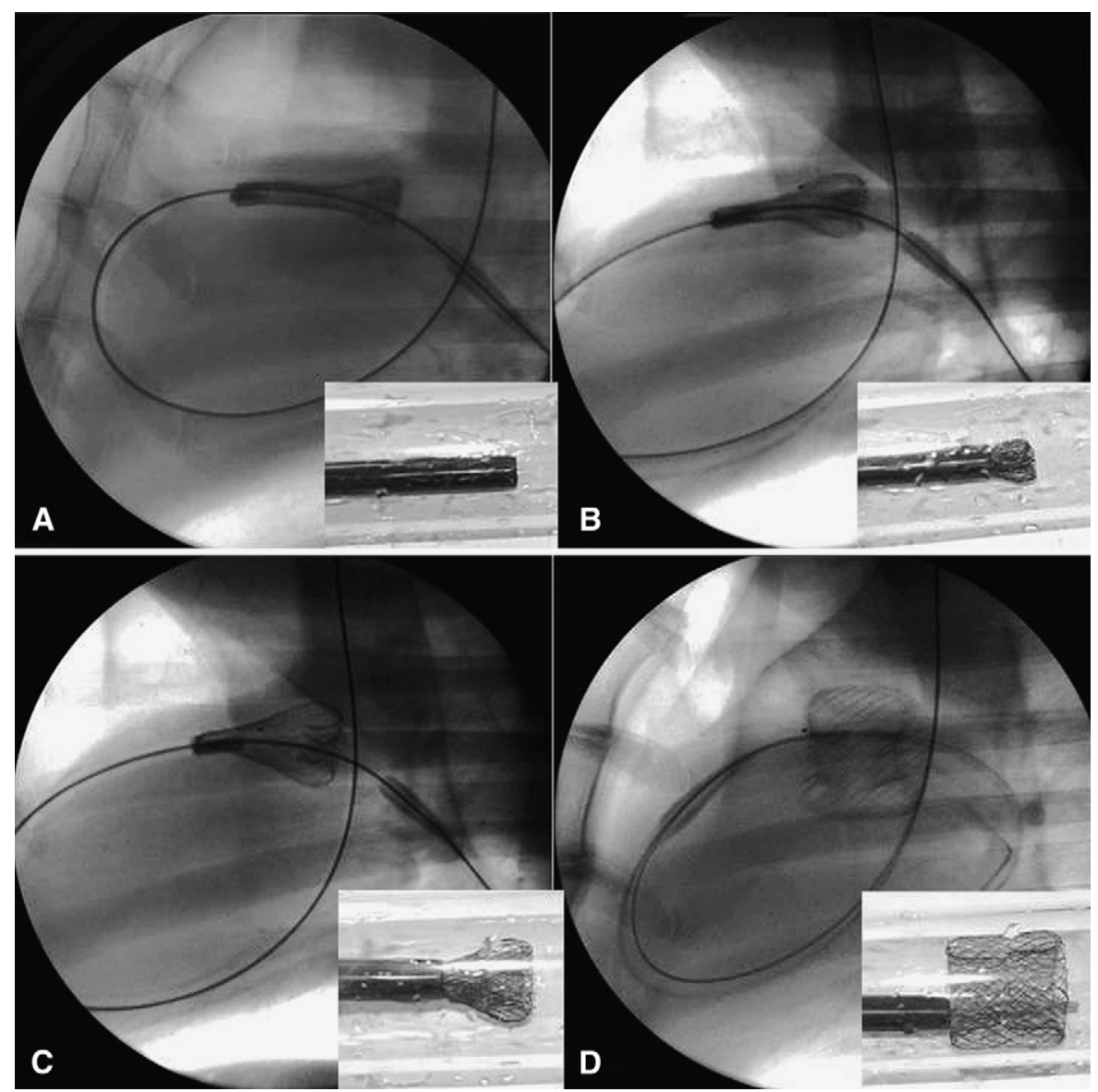

FIGURE 3. Angiographic views showing the delivery of the PA filler. In each panel (A-D) an in vitro photograph is provided, showing the opening of the device in a plastic tube.

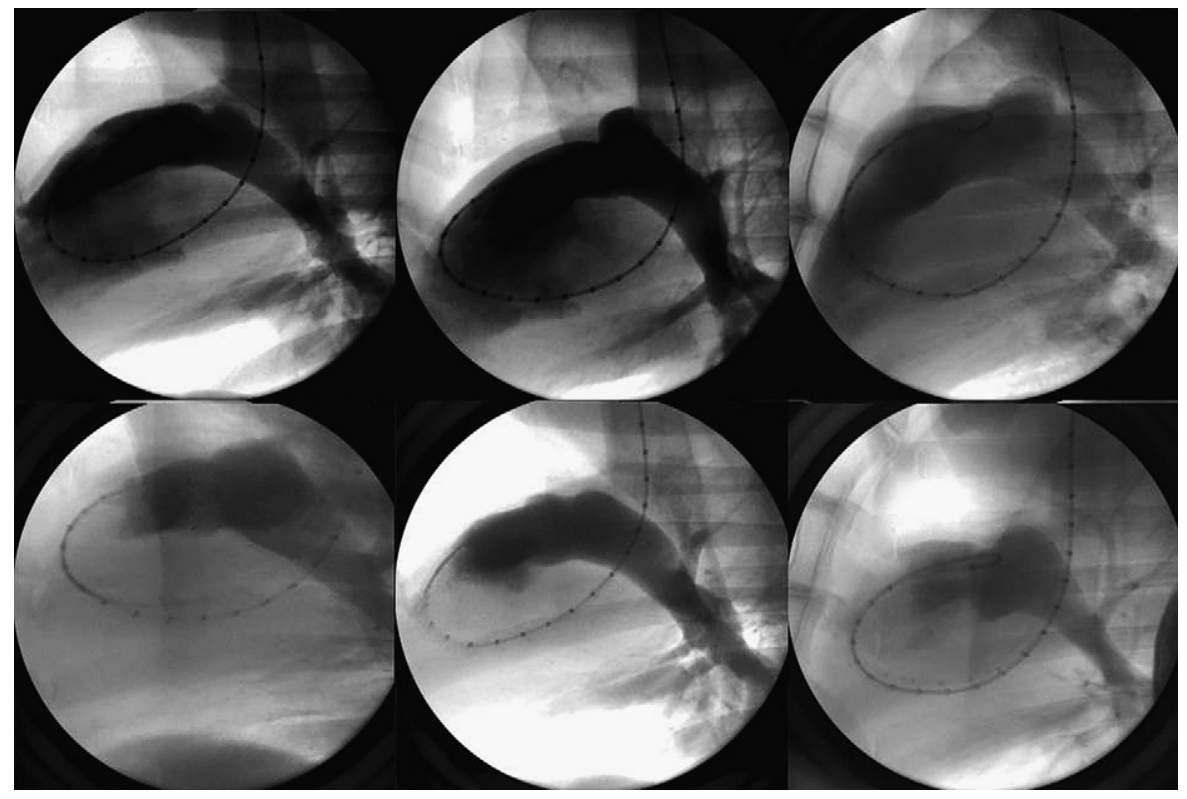

FIGURE 4. Angiograms before insertion of the filler showing the variable anatomy of the RVOT obtained with patch enlargement. 
TABLE 1. Characteristics of animals from group 1

\begin{tabular}{|c|c|c|c|c|c|c|c|c|}
\hline Group 1 & $\begin{array}{l}\text { MPA circumference } \\
\text { after surgery }(\mathbf{m m})\end{array}$ & $\begin{array}{c}\text { MPA diameter } \\
\text { after surgery }(\mathbf{m m})\end{array}$ & $\begin{array}{l}\text { MPA diameter at } \\
\text { follow-up (mm) }\end{array}$ & $\begin{array}{c}\text { Time between surgery } \\
\text { and insertion of filler (d) }\end{array}$ & $\begin{array}{c}\text { RV } \\
\text { dilation* }\end{array}$ & $\begin{array}{c}\text { RV } \\
\text { failure* }\end{array}$ & PVI & $\begin{array}{l}\text { Filler } \\
(\mathrm{mm})\end{array}$ \\
\hline 1 & 110 & 35 & 34 & 0 & - & - & - & $40 \dagger$ \\
\hline 2 & 120 & 38.2 & 35 & 94 & 1 & 0 & Severe & $40 \dagger$ \\
\hline 3 & 115 & 36.6 & 31 & 86 & 0 & 0 & Moderate & 35 \\
\hline 4 & 110 & 35 & 30 & 115 & 1 & 1 & Severe & 35 \\
\hline 5 & 108 & 34.4 & 33 & 100 & 0 & 0 & Severe & 35 \\
\hline 6 & 120 & 38.2 & 36 & 70 & 1 & 0 & Severe & 40 \\
\hline Mean & 113.8 & 36.2 & 33.2 & 77.5 & $3 / 5$ & $1 / 5$ & NA & NA \\
\hline
\end{tabular}

$M P A$, Main pulmonary artery; $R V$, right ventricular; $P V I$, pulmonary valve incompetence; $N A$, not applicable. ${ }^{*} 1$, present; 0 , absent. $\dagger$ Transventricular insertion of the filler.

crossclamp was being released, and another had a refractory ventricular arrhythmia during crossclamping of the RVOT. The last one died at the time of the second stage during anesthetic induction. Autopsy showed a severely enlarged $\mathrm{RV}$ with pleural effusion. In the remaining 30 animals, no complication occurred from the initial operation to the second stage. One animal from group 1 had a cardiac arrest at induction but was successfully resuscitated. The mean baseline diameter of the PA was $25.3 \mathrm{~mm}$, ranging from
19.1 to $29.2 \mathrm{~mm}$. This mean diameter was augmented with patch enlargement to $36.2 \mathrm{~mm}$ (range, 31.8-40 $\mathrm{mm}$ ). Valvular excision was possible in 31 of 33 animals. In 2 cases, the leaflet was not caught in the vascular clamp. In those, we tried to suture the leaflet from the outside after patch suture and release of the clamp. We were successful in only 1 of the 2 . As a result, $96 \%$ of the animal had pulmonary regurgitation. The recovery period lasted a mean of 89 days (range, 60-191 days). The anatomy of the enlarged

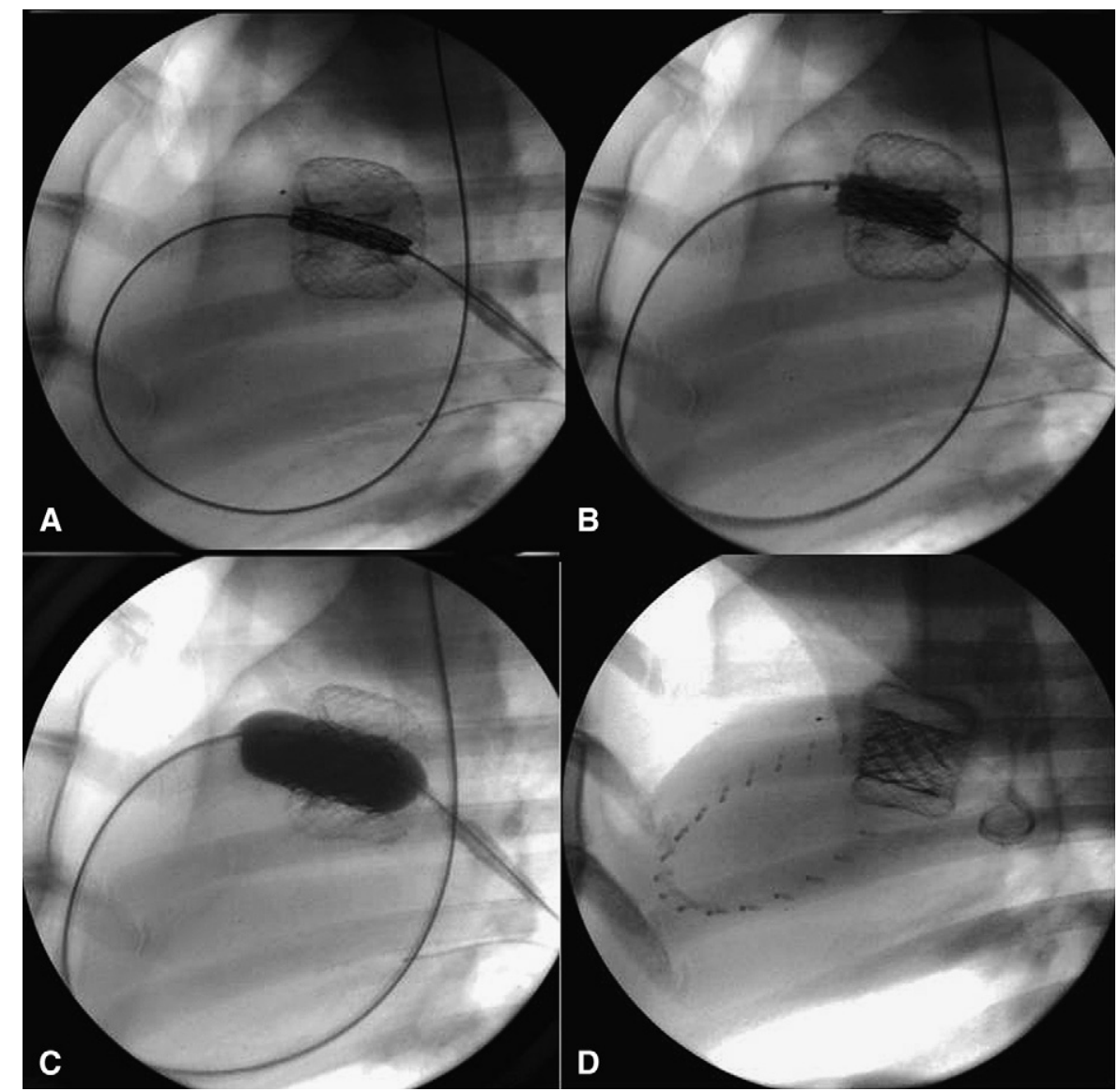

FIGURE 5. Angiograms showing the insertion of the valved stent inside the PA filler. A, Placement of the valved stent. B, Inflation of the inner balloon catheter. C, Inflation of the outer balloon catheter. D, Aspect after full deployment of devices. 
RVOT was variable despite similar surgical technique. Examples are given in Figure 4. The angiographic diameter of the PA was smaller, varying from 30 to $40 \mathrm{~mm}$ (mean, $33.3 \mathrm{~mm}$ ).

\section{Animals from Group 1: Feasibility Group}

The filler could be deployed and successfully placed in the enlarged part of the PA in all animals (Table 1). Implantation was made transventricularly in the first 2 animals and percutaneously in the other. The transventricular approach was chosen electively in 1 animal and after unsuccessful advancement of the delivery system in another. Both insertions were successful. After the animals were humanely killed, macroscopic study confirmed the angiographic statements showing the correct deployment, location, and impaction of the devices on the vascular wall.

\section{Animals from Group 2}

Animals $(n=12)$ successfully received the filler and subsequently the PV via the transvenous approach (Figure 5 and Table 2). Angiograms confirmed the good deployment and placement of the devices away from the PA bifurcation. No regurgitation or leak was noticed in 9 of 12 animals. In those, dye injection in the RV did allow visualization of the aneurysmal RVOT. The filler-valve assembly was perfectly molded in all (Figure 6). Respectively, 2 and 1 animals from groups $2 \mathrm{~A}$ and $2 \mathrm{~B}$ had a significant paraprosthetic leak (Figure 7). In the animal from group 2B, the valved stent was placed too far proximally, allowing a persisting gap between the inner part of the filler and the extremity of the valved stent. Oversizing of the filler fully explained the gap, making insertion of an $8 \mathrm{Z} 28$ stent too short to cover it. This leak was treated by the insertion of a longer valved stent (8Z34). In the remaining 2 animals, the paraprosthetic leak was important, almost completely filling the aneurysmal RVOT. Excluding animals with leak, the systolic gradient across the devices was as low as a mean of $7 \mathrm{~mm} \mathrm{Hg}$ (range, $4-11 \mathrm{~mm} \mathrm{Hg}$ ). All animals survived to follow-up. All devices were in good position. No embolization occurred. At 1 month (mean, 32.7; range, 28-42 days), the mean transprosthetic gradient was stable at $6 \mathrm{~mm} \mathrm{Hg}$ (range, $0-10 \mathrm{~mm} \mathrm{Hg}$ ). The paraprosthetic leak from the 2 animals (group 2A) was unchanged and none appeared in the other animals. At autopsy, all devices were almost completely fixed to the PA, making their retrieval impossible. One PTFE membrane was broken, which explained the leak seen in this animal. In the second animal with a leak, the filler was not against the anterior wall of the PA. At this level, the filler was not fixed to the PA. A fresh thrombus was present, fully indicating the location of the leak suspected on angiograms. Entry and exit points were at the same level. As in others, retrieval of the filler from the PA was not possible. All valvular implants were thin and mobile.

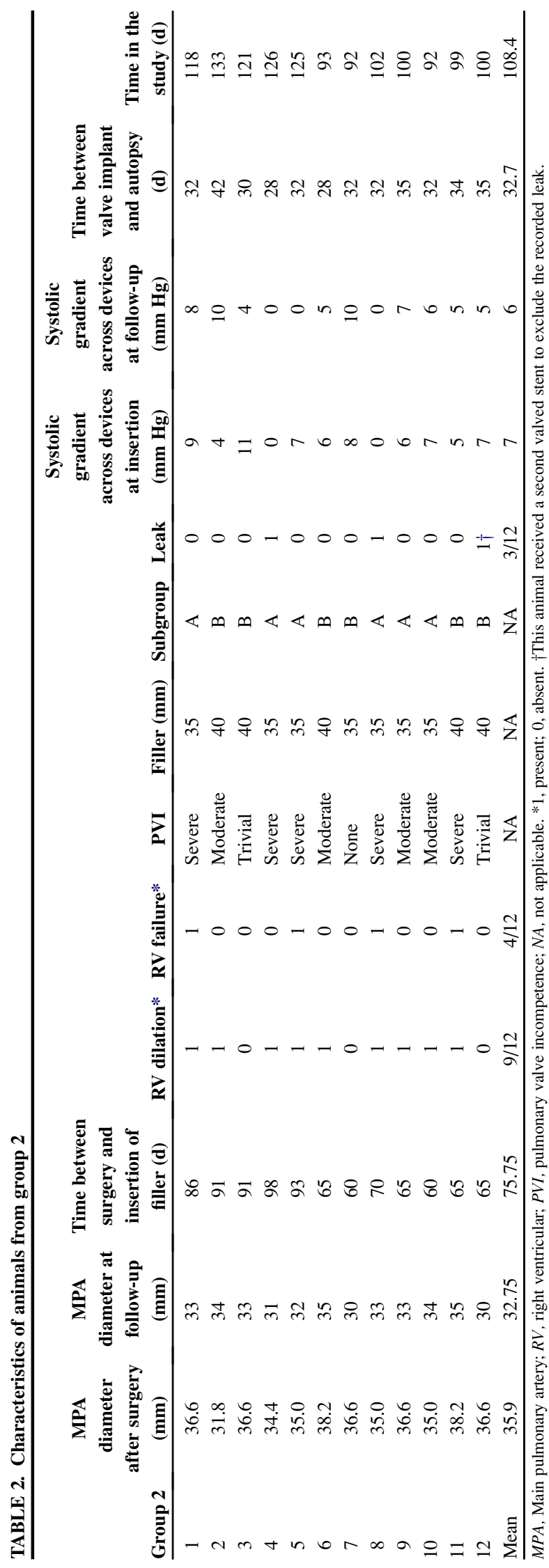




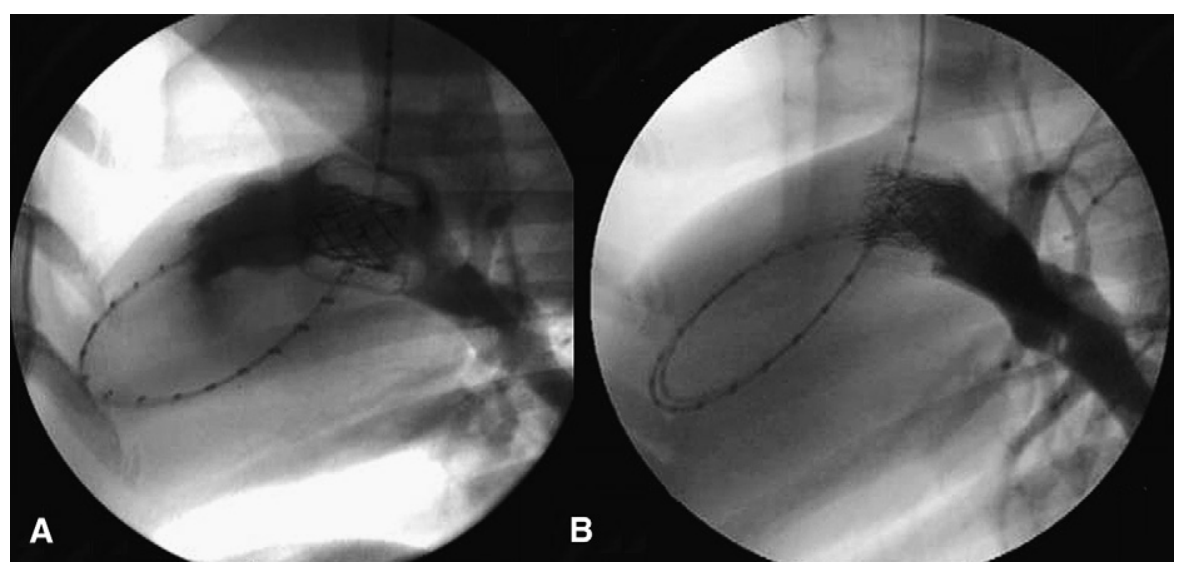

FIGURE 6. Angiograms after dye injection showing the function of implanted devices (equivalent of a lateral view). A, Right ventriculogram showing the perfect sealing of the device and the reduction of PA diameter. B, PA injection showing good function of the implanted valve.

\section{Animals from Group 3}

The transvenous insertion of the PA filler was possible in all (12 of 12). In 1 animal, because no larger device was available, a $40-\mathrm{mm}$ filler was implanted in a $40-\mathrm{mm}$ PA (animal initially allocated to group 3B but reallocated to group 3A). During retrieval of the delivery system, the device migrated next to the PA bifurcation. We decided not to proceed with valve insertion and the animal was humanely killed. Except in this animal, all fillers were perfectly stable and a valved stent was subsequently inserted. In one instance, a defective stent burst the balloon at the end of the inflation, making its deflation impossible. The animal died before we could retrieve the delivery catheter (group 3B). In the remaining 10 animals, the procedure was uneventful (Table 3). Angiograms showed good deployment and position of the devices, but a paraprosthetic leak was present in 3 of 10 . In 1 animal, the leak was moderate and present only on the PA injection and not on the RV in- jection ("distal leak"); the dye washout was very slow. All animals were from group 3A. Excluding these animals, the mean transprosthetic gradient was $6 \mathrm{~mm} \mathrm{Hg}$ (range, 1-10 $\mathrm{mm} \mathrm{Hg}$ ).

All animals (excluding the animal undergoing unsuccessful implantation) survived to follow-up. At 1 and 2 months of follow-up, no embolization occurred. One animal died at anesthetic induction and could not be tested at 2 months' follow-up. At autopsy, the valved stent was severely obstructed by valvular calcifications. In tested animals, the transprosthetic gradient remained in the same range at the two evaluations: $7 \mathrm{~mm} \mathrm{Hg}$ (range, $4-8 \mathrm{~mm} \mathrm{Hg}$ ) and $5 \mathrm{~mm}$ Hg (range, 0-8), respectively. A paraprosthetic leak was present in the same 3 animals. It was trivial to moderate in the animal with the distal leak (ie, leak seen only on the pulmonary angiogram). Autopsy was performed after a mean follow-up of 8.9 weeks (range, 7-16 weeks). Because of a scheduling problem, 1 animal was humanely killed at 16
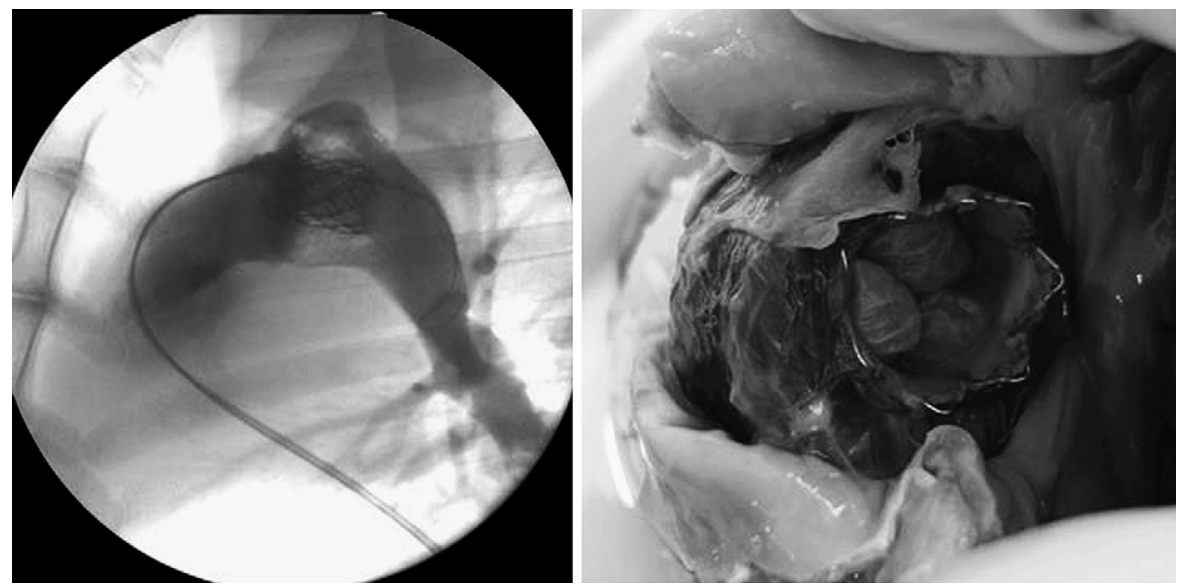

FIGURE 7. Left, Angiograms showing the RVOT after insertion of the devices in an animal with an undersized filler. Note the significant paravalvular leak represented by the presence of contrast dye around the filler. Right, The aspect of the RVOT at autopsy after opening of the anterior wall. The filler is not embedded in the anterior part of the RVOT, which completely explains the leak seen on angiograms. 


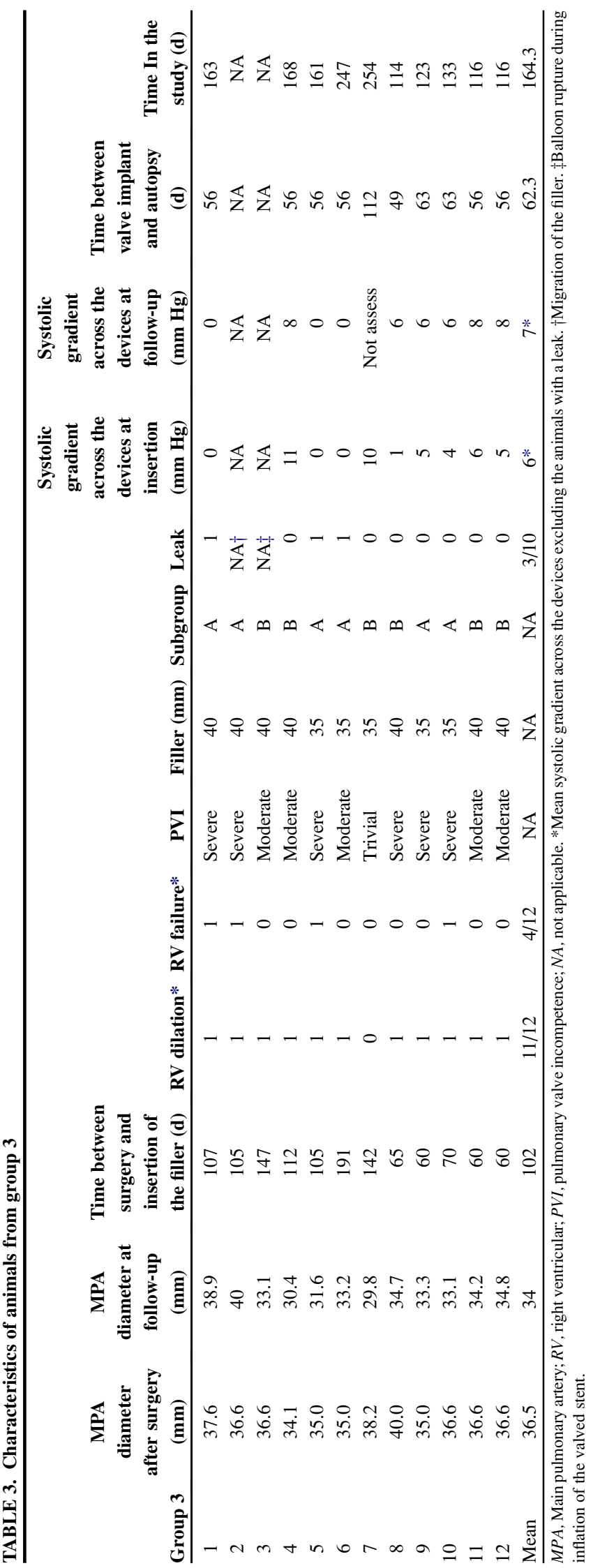

weeks. Endothelialization of devices was complete. Devices were completely embedded in the vascular wall and impossible to retrieve. Fillers and valved stents formed one unique block. Twice, a fixation defect was present anteriorly or posteriorly, fully explaining the leak seen on RV and PA angiograms. The last leak was due to a small hole in the PTFE membrane on the distal part of the filler ("distal leak"). No fixation defect was found in this animal. Valve implants (except the one described previously) were thin and mobile in all animals.

\section{Histologic Study}

Histologic findings were similar in all animals (Figures 8 and 9). A fibroblastic proliferation was noticed between the nitinol stent and vascular wall. Few inflammatory cells were present close to the PTFE membrane and especially in connection with the threads. No necrotic area was noticed. In addition, a fibroblastic proliferation was found within the middle part of the nitinol stent. Loose connective tissue was finally observed between the nitinol and valved stents except in the area in contact with PTFE membrane, where a thin and dense layer of fibroblasts was present. Valve implants appeared normal in every case except in the animal with macroscopic calcifications. Elsewhere, no inflammatory infiltrate or calcifications were present (Figure 9, D).

\section{DISCUSSION}

Transcatheter PV insertion technique is still inaccessible to the wide majority of patients who have a native RVOT exceeding $22 \mathrm{~mm}$ in diameter. We intended to test a device that intravascularly reduces the diameter of the PA and permits subsequent insertion of a commercially available valved stent. Although feasibility and anchorage were demonstrated in a previous study, the design had to be improved to facilitate the deployment. ${ }^{18,19}$ Moreover, the anchorage properties of the filler had to be tested in wide and asymmetrical RVOTs. We imagined an animal model with enlarged RVOTs. Thirty-three animals were operated on and had PA enlargement with a pericardial patch and the creation of a pulmonary leak. Three animals died after this operation. Significant PVI was achieved in the great majority, leading to dilation of the RV. As expected, the PA was enlarged with a mean diameter of $36.2 \mathrm{~mm}$.

\section{Insertion of the Filler and Subsequent Complications}

All fillers were inserted successfully. The modifications made to the configuration of the device improved its deployment and placement as compared with a previous study. ${ }^{19}$ No extra handling was needed to release the filler. We encountered only one embolization over 30 implants. This occurred suddenly in an animal in which we implanted a device with a diameter equal to the diameter of the enlarged PA. Besides this embolization, we faced a paraprosthetic leak in $25 \%$ of the animals. The leak was 


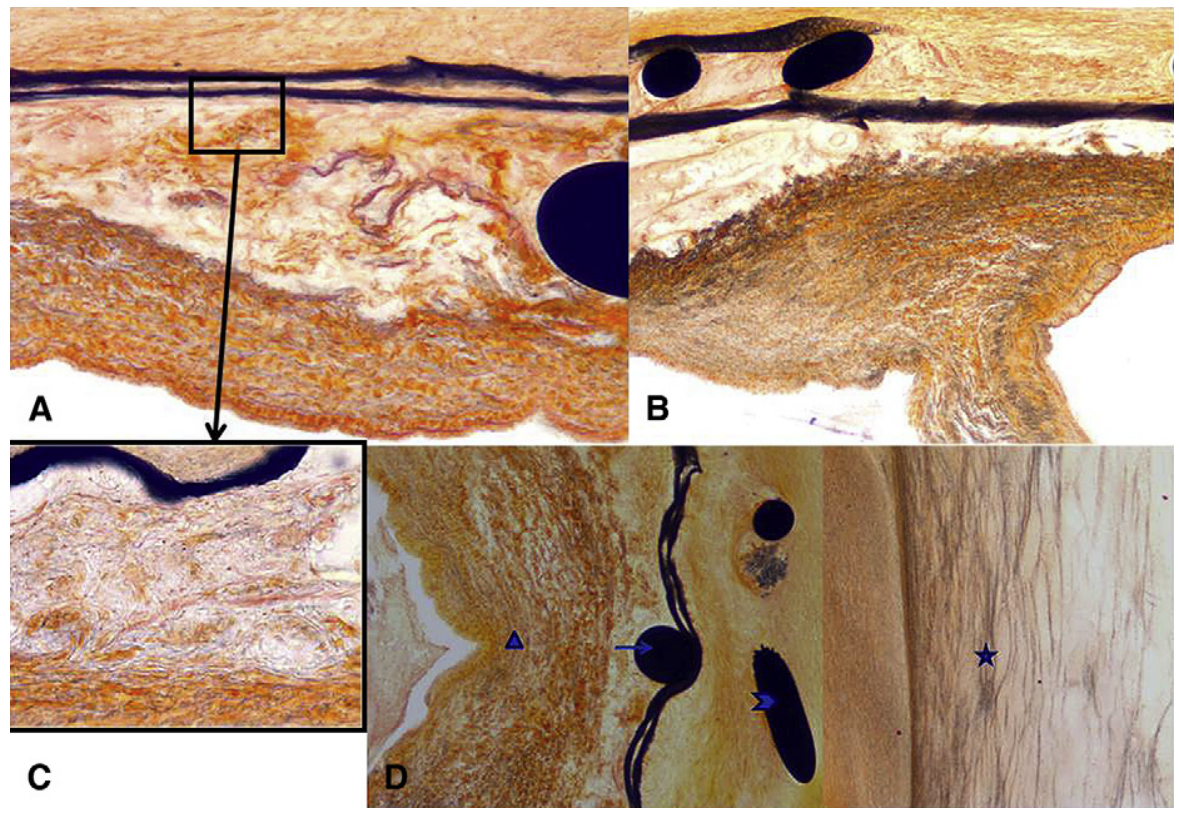

FIGURE 8. Histologic views (trichrome). A and B, Loose connective tissue between the nitinol (black circle on the right) and valved stents (trichrome $\times 50$ ). $\mathrm{C}$, Magnification of panel A (trichrome $\times 100$ ). D, Overview of the vascular wall (star) with the nitinol stent (arrowhead), valved stent (arrow), and bovine arterial wall (triangle).

related to undersizing of the device. Indeed, $5(83 \%)$ of the 6 leaks occurred in animals in which the size of the device was less than $5 \mathrm{~mm}$ greater than the PA diameter $(P<.05)$. This confirms that oversizing the device is mandatory for stent anchoring and ultimately for definitive fixation to the vascular wall. Although mandatory, oversizing should not be excessive. In 1 animal, the device was oversized by $10 \mathrm{~mm}$, resulting in a stable but less than fully deployed
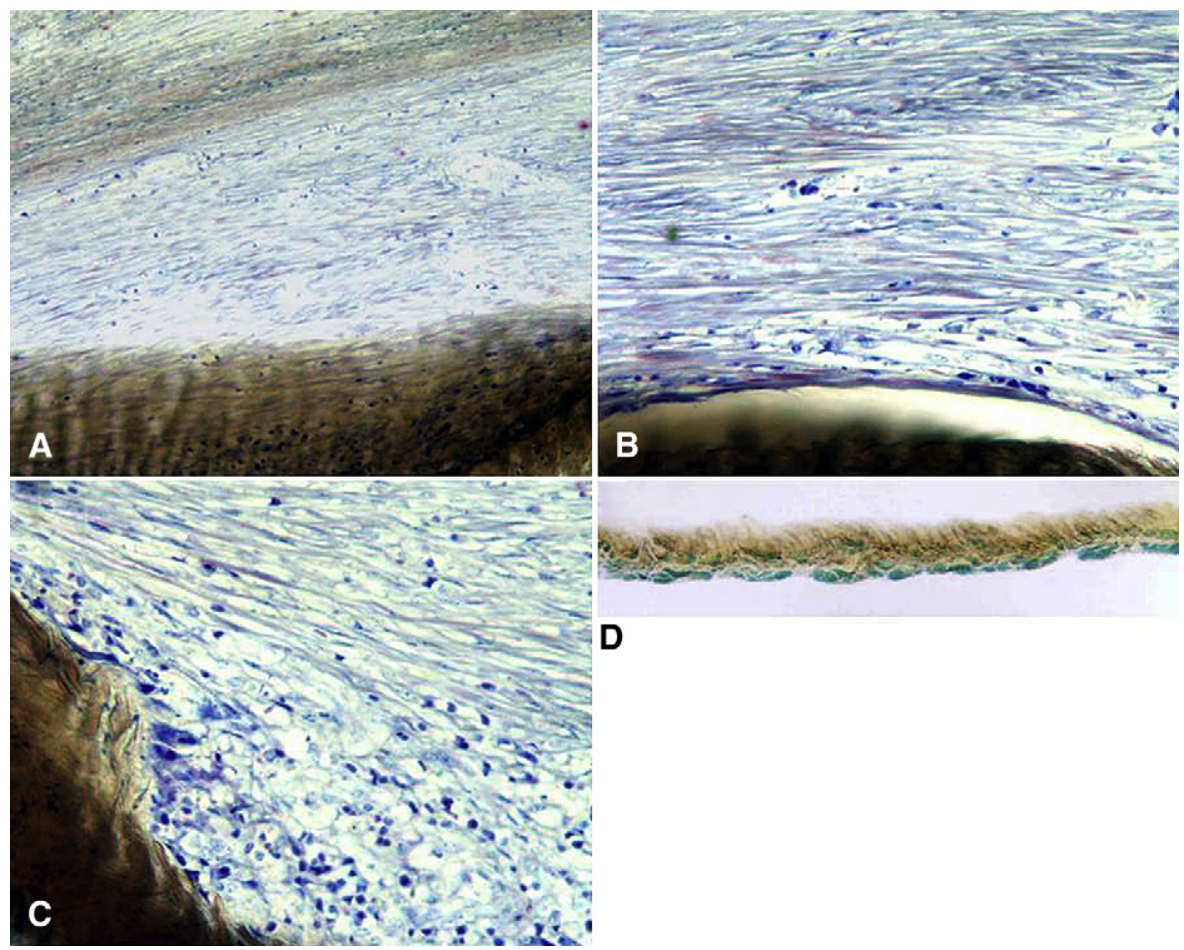

FIGURE 9. Histologic views (Stevenel Blue). A, Dense connective tissue between the nitinol stent and the arterial wall $(\times 100)$. B and C, Mild inflammatory infiltrate between the nitinol stent and the arterial wall $(\times 200)$. D, Valve implant with normal thickness $(\times 50)$. 
device. As a consequence, the space between the extremities in the valve receiving part of the filler was enlarged. This complication was not anticipated, and the inserted valved stent was too short to cover the entire gap, leading to the insinuation of blood between the two devices. This leak was further excluded by insertion of a longer valved stent. PTFE membrane breaks were also a source of paraprosthetic leak in 2 animals (of 6 paraprosthetic leaks).

\section{PV Insertion}

PV replacement was possible in 28 of 29 animals without any complication. In 1 animal, the balloon ruptured during its inflation because of a defective valved stent. Its deflation was impossible and the animal died before the delivery system could be retrieved. Excluding this animal, insertion was uneventful and easily done without the need for contrast dye injection. As previously shown, hemodynamic studies performed immediately and during follow-up did not show a significant RV-PA gradient in the majority of animals. One animal with a calcified valve and probable elevated $\mathrm{RV}$ pressure could not be assessed because it died at induction before evaluation.

\section{Limitations to the Study and Unanswered Questions}

Even if the model offers a new feature to test devices for large RVOTs, the obtained anatomy is still very different from that seen in postoperative Fallot patients in need of PV replacement. However, we were able to create a good model of an enlarged and asymmetrical RVOT and PVI. The plasticity of the device was very good, making it adequate to conform to all created RVOT anatomy. Whether this would apply to human anatomy with calcified or noncalcified RVOT and with noncircumferential cross-sectional shape remains to be proven.

The described animal model led to PVI. As a result, animals had significant RV dilatation (77\%) and/or dysfunction $(30 \%)$. Unfortunately, these features were assessed subjectively with angiograms and hemodynamic data. A more quantitative way, such as magnetic resonance imaging, to assess this dysfunction would be very useful, but this imaging modality is not presently available in our animal laboratory.

Although we were able to show that oversizing is preferable in animals, this could be very different in humans and will need proper studies. In our study, the simple measurements of the maximum diameter of the PA were sufficient to choose the proper device. In the clinical setting, a more sophisticated approach such as the use of compliant balloon catheters might be useful to improve device selection. In addition, having a device in the RVOT might, at least theoretically, increase the risk of thrombus formation. Even if we did not observe such a complication in animals, a protocol of prophylaxis with antithrombotic agents will need to be refined in humans. Finally, despite considerable improve- ments in stent design, a more direct procedure would be preferable. We are, indeed, in the process of developing an integrated valved stent that could be inserted in one step in the enlarged PA.

\section{CONCLUSIONS}

The realization of an ovine model of a large and asymmetrical RVOT was possible with a small learning curve. We were able to reproduce the RVOT enlargement with a patch and the presence of a pulmonary regurgitation with repercussions on the RV. The deployment of the new design of the filler was possible in the vast majority of animals. The choice of device size is critical to avoid complications such as embolization and paraprosthetic leak.

We thank Philippe Marx, who developed this new stent in cooperation with Younes Boudjemline. We also thank Professor D. Hannouche and Mr Myrtil for their technical support in the histologic studies.

\section{References}

1. Nollert G, Fischlein T, Bouterwek S, Böhmer C, Klinner W, Reichart B. Longterm survival in patients with repair of tetralogy of Fallot: 36-year follow-up of 490 survivors of the first year after surgical repair. J Am Coll Cardiol. 1997;30: 1374-83.

2. Murphy JG, Gersh BJ, Mair DD, Fuster V, McGoon MD, Ilstrup DM, et al. Longterm outcome in patients undergoing surgical repair of tetralogy of Fallot. $N$ Engl J Med. 1993;329:593-9.

3. Hamada H, Terai M, Jibiki T, Nakamura T, Gatzoulis MA, Niwa K. Influence of early repair of tetralogy of Fallot without an outflow patch on late arrhythmias and sudden death: a 27-year follow-up study following a uniform surgical approach. Cardiol Young. 2002;12:345-51.

4. Silka MJ, Hardy BG, Menashé VD, Morris CD. A population-based prospective evaluation of risk of sudden cardiac death after operation for common congenital heart defects. J Am Coll Cardiol. 1998;32:245-51.

5. Gatzoulis MA, Till JA, Somerville J, Redington AN. Mechanoelectrical interaction in tetralogy of Fallot. QRS prolongation relates to right ventricular size and predicts malignant ventricular arrhythmias and sudden death. Circulation. 1995; 92:231-7.

6. Deanfield JE, McKenna WJ, Presbitero P, England D, Graham GR, HallidieSmith K. Ventricular arrhythmia in unrepaired and repaired tetralogy of Fallot Relation to age, timing of repair, and haemodynamic status. Br Heart J. 1984; 52:77-81.

7. Dietl CA, Cazzaniga ME, Dubner SJ, Pérez-Baliño NA, Torres AR, Favaloro RG Life-threatening arrhythmias and RV dysfunction after surgical repair of tetralogy of Fallot. Comparison between transventricular and transatrial approaches. $\mathrm{Circu}$ lation. 1994;90(5 Pt 2):II7-12.

8. Hokanson JS, Moller JH. Significance of early transient complete heart block as a predictor of sudden death late after operative correction of tetralogy of Fallot. Am J Cardiol. 2001;87:1271-7.

9. Bove EL, Kavey RE, Byrum CJ, Sondheimer HM, Blackman MS, Thomas FD. Improved right ventricular function following late pulmonary valve replacement for residual pulmonary insufficiency or stenosis. J Thorac Cardiovasc Surg. 1985;90:50-5.

10. Bove EL, Byrum CJ, Thomas FD, Kavey RE, Sondheimer HM, Blackman MS et al. The influence of pulmonary insufficiency on ventricular function following repair of tetralogy of Fallot: evaluation using radionuclide ventriculography. $J$ Thorac Cardiovasc Surg. 1983;85:691-6.

11. Harrison DA, Harris L, Siu SC, MacLoghlin CJ, Connelly MS, Webb GD, et al. Sustained ventricular tachycardia in adult patients late after repair of tetralogy of Fallot. J Am Coll Cardiol. 1997;30:1368-73.

12. de Ruijter FT, Weenink I, Hitchcock FJ, Meijboom EJ, Bennink GB. Right ventricular dysfunction and pulmonary valve replacement after correction of tetralogy of Fallot. Ann Thorac Surg. 2002;73:1794-800. 
13. Bonhoeffer P, Boudjemline Y, Saliba Z, Hausse AO, Aggoun Y, Bonnet D, et al. Transcatheter implantation of a bovine valve in pulmonary position: a lamb study. Circulation. 2000;102:813-6.

14. Bonhoeffer P, Boudjemline Y, Qureshi SA, Le Bidois J, Iserin L, Acar P, et al. Percutaneous insertion of the pulmonary valve. J Am Coll Cardiol. 2002;39:1664-9.

15. Lurz P, Coats L, Khambadkone S, Nordmeyer J, Boudjemline Y, Schievano S, et al. Percutaneous pulmonary valve implantation: impact of evolving technology and learning curve on clinical outcome. Circulation. 2008;117:1964-72.

16. Berdat PA, Carrel T. Off-pump pulmonary valve replacement with the new Shelhigh Injectable Stented Pulmonic Valve. J Thorac Cardiovasc Surg. 2006;131:1192-3.
17. Schreiber C, Vogt M, Bauernschmitt R, Prodan Z, Tassani P, Eicken A, et al. Pulmonary valve implantation with a self-expanding stented valve: 1-year follow-up in the first patient. Ann Thorac Surg. 2006;82:2340.

18. Boudjemline Y, Agnoletti G, Bonnet D, Sidi D, Bonhoeffer P. Percutaneous pulmonary valve replacement in a large right ventricular outflow tract: an experimental study. J Am Coll Cardiol. 2004;43:1082-7.

19. Mollet A, Basquin A, Stos B, Boudjemline Y. Off-pump replacement of the pulmonary valve in large right ventricular outflow tracts: a transcatheter approach using an intravascular infundibulum reducer. Pediatr Res. 2007;62: 428-33. 